\title{
The many forms of beta diversity: a comment on McGill et al. and some notational suggestions
}

\author{
Tobias Jeppsson ${ }^{1,2}$
}

\begin{abstract}
Affiliations:
${ }^{1}$ Department of Ecology, The Swedish University of Agricultural Sciences, Uppsala, Sweden

${ }^{2}$ Centre of Ecological and Evolutionary Synthesis, University of Oslo, Norway
\end{abstract}

Corresponding author: Jeppsson, T. (tobias.jeppsson@slu.se).

Keywords: Biodiversity change, $\beta$-diversity, community ecology

Fundamentally, beta diversity is a measure of species turnover across time or space. In practice, it is sometimes unclear exactly what aspect of beta diversity that is implied in studies. For instance, a trend in 'spatial beta diversity' can be used to refer to both differences in spatial beta diversity between sites, as well as a temporal trend in spatial beta diversity (at the same site). In a recent review, McGill et al.[1] provide a useful and much needed overview of different aspects of biodiversity change, and show areas where we lack knowledge. Even so, McGill et al. ignore some aspects of beta diversity and sometimes pool different types of beta diversity under the same heading. However, their review mainly focused on temporal trends in diversity, while I here want to highlight spatial patterns in temporal $\beta$-diversity (species turnover) as an important but somewhat overlooked component of biodiversity change. Furthermore, I propose a slightly modified classification and nomenclature of metrics of biodiversity change, with the aim of complementing their review. The notation used here can hopefully be useful to other authors as well.

To create their matrix of 15 forms of biodiversity trends, McGill et al.[1] use 4 classes of biodiversity metrics ( $\alpha$ diversity, spatial $\beta$ diversity, temporal $\beta$ diversity and abundance), but these classes each contain different aspects of biodiverstiy change and are therefore not as clearcut as they may appear. I suggest that there are at least eight key aspects to biodiversity change from the perspective of species richness; $\alpha \vec{s}, \alpha_{\vec{t}}, \beta_{t}, \beta_{s}, \beta_{t, \vec{s}}, \beta_{s, \vec{t}}, \beta_{t, \vec{t}}$ and $\beta_{s, \vec{s}}$, to which we can add measures of abundance or biomass (see Fig. 1). Here, $s$ and $t$ refer to spatial or temporal dimensions and $\vec{s}$ and $\vec{t}$ indicate change across space or time.

The two aspects of alpha diversity are relatively straightforward, and describe temporal and spatial patterns of local species richness. The six aspects of beta 


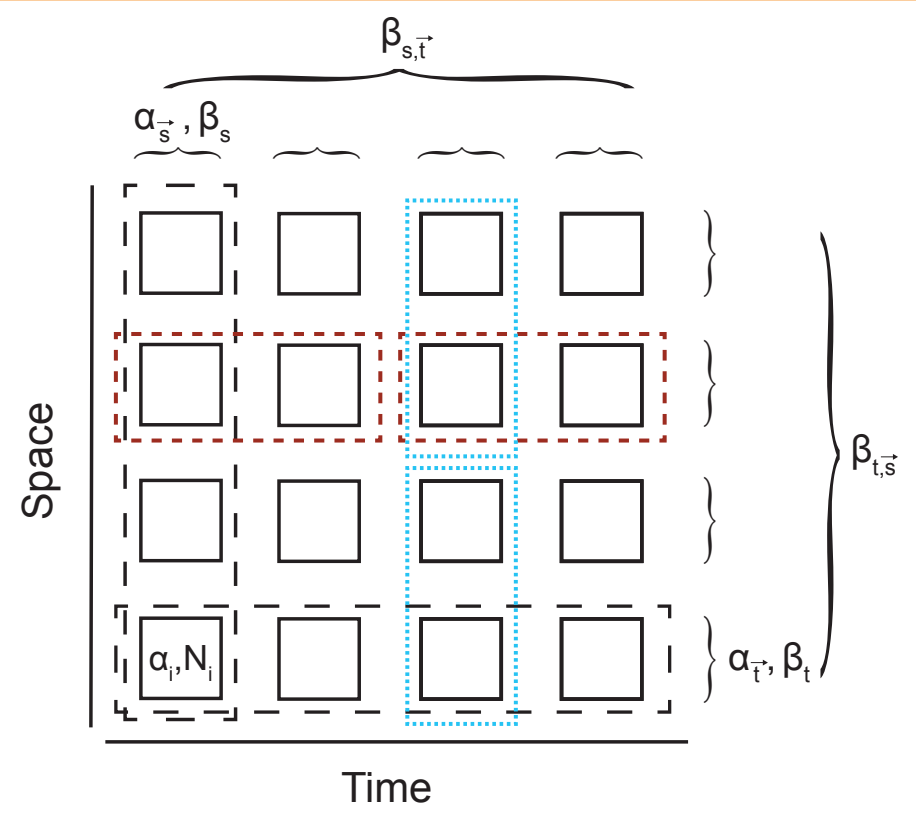

Figure 1: Schematic view of aspects of biodiversity change along the dimensions of time and space, focusing on $\beta$-diversity. Squares represent units of analysis (sites), the dashed black lines indicate measures across units and large brackets indicate patterns across dimensions. Dashed red lines indicate a grouping that corresponds to $\beta_{t, \vec{t}}$ and dotted blue lines indicate a grouping that corresponds to $\beta_{s, \vec{s}}$. In all indices of biodiversity, $\mathrm{s}$ and $\mathrm{t}$ refer to spatial or temporal dimensions and $\vec{s}$ and $\vec{t}$ indicate change across space or time.

diversity are more interesting in relation to the results presented in McGill et al.[1], where $\beta_{t, \vec{s}}$ is not discussed. In the review of empirical results it is also clear that they do not focus on spatial $\beta$-diversity directly $\left(\beta_{s}\right)$, but rather temporal trends in spatial $\beta$-diversity $\left(\beta_{s, \vec{t}}\right)$, while $\beta_{t}$ is presented on its own terms, especially with regard to estimates of average turnover rates across studies. Note that the six measures of beta diversity presented above represents two corresponding pairs, where $\beta_{t}$ and $\beta_{s}$ are static measures of $\beta$-diversity at a particular dimension (space or time) and scale. Both betas are also used to measure the degree of similarity (or similarity decay) over either time or space. One the other hand, $\beta_{t, \vec{s}}, \beta_{s, \vec{t}}, \beta_{t, \vec{t}}$ and $\beta_{s, \vec{s}}$ all show dynamic patterns across space or time. As explained in McGill et al., changes in $\beta_{s, \vec{t}}$ describe the degree of biotic homogenization (or diversification) over time at a particular spatial scale. In parallel, patterns in $\beta_{t, \vec{s}}$ indicate whether rates of temporal species turnover differ in systematic ways between sites. For completeness, you can also add $\beta_{t, \vec{t}}$ and $\beta_{s, \vec{s}}$ (Fig. 1), which could refer to temporal trends in species turnover and differences in spatial $\beta$-diversity between land-use types respectively, where the former is mentioned in McGill et al.[1] in reference to regime shifts in aquatic environments and the latter with respect to types of coffee plantations.

Even if spatial patterns or trends in temporal species turnover $\left(\beta_{t, \vec{s}}\right)$ might seem esoteric, they are highly relevant for many issues in ecology and conservation biology. For instance, large-scale latitudinal patterns in $\beta_{t}$ might help us to 
understand well-known macroecological patterns such as the latitudinal gradient in species richness[2]. With ongoing climate change, gradients of $\beta_{t, \vec{s}}$ that runs north-south or across elevation levels are important to understand, since this can influence how communities respond to the effects of warming. Finally, patterns of $\beta_{t, \vec{s}}$ can be compared to local or regional site characteristics, to understand if e.g. overall site productivity or the type of land-use is influencing the temporal turnover of species.

There are of course good reasons for why $\beta_{t}, \vec{s}$ has received relatively little attention, compared to other aspects of $\beta$-diversity. The main reason is probably the general lack of comparable, high quality, long term studies of biodiversity, which are needed to study spatial patterns in temporal species turnover. To be clear, I'm not proposing anything new here, and aspects of $\beta_{t, \vec{s}}$ has been addressed in earlier studies (as I'm sure the authors of McGill et al. are well aware), for instance in Russell et al.[3] and White et al.[4], and the type of data presented in Dornelas et al.[5] could also be used to this end. To conclude, I fully support the call in McGill et al.[1] for an increased focus on aspects of $\beta$-diversity, and hope that $\beta_{t, \vec{s}}$ will be recognized more clearly in studies of biodiversity change, even if we currently have few empirical studies that address this issue, especially at larger spatial scales.

\section{Acknowledgements}

I want to thank The Swedish Forest Society for funding my research and T. Pärt, J. Bengtsson, J. Knape and M. Hiron for helpful remarks on earlier versions of this text.

\section{References}

1 McGill, B.J. et al. (2015) Fifteen forms of biodiversity trend in the Anthropocene. Trends in Ecology \& Evolution 30, 104-113

2 Gaston, K.J. et al. (2000) Global patterns in biodiversity. Nature 405, $220-227$

3 Russell, G.J. et al. (1995) A Century of Turnover: Community Dynamics at Three Timescales. Journal of Animal Ecology 64, 628-641

4 White, E.P. et al. (2006) A comparison of the species-time relationship across ecosystems and taxonomic groups. Oikos 112, 185-195

5 Dornelas, M. et al. (2014) Assemblage Time Series Reveal Biodiversity Change but Not Systematic Loss. Science 344, 296-299 